\title{
Neumotórax espontáneo sintomático en un recién nacido: reporte de caso
}

\section{Symptomatic spontaneous pneumothorax in a newborn: a case report}

Jaime Alberto Urbiña Villarraga, ${ }^{1}$ María Catalina Medina Escobar, ${ }^{2}$ Carlos Julián Contreras Merchan ${ }^{3}$ Diego Alejandro Torres Gómez ${ }^{4}$

\section{Resumen}

El neumotórax espontáneo sintomático es un evento poco frecuente en el recién nacido. Aunque su curso es benigno y con resolución espontánea en la mayoría de los casos, en algunos se deben realizar intervenciones más agresivas. En la literatura colombiana no hay registro de un caso de neumotórax espontáneo en recién nacido, ni estadísticas completas de eventos similares de escapes aéreos.

Se presenta el reporte de caso de una recién nacida a término, con parto eutócico y buena adaptación al momento del nacimiento, quien presenta con el transcurso de las horas episodio de dificultad respiratoria severa que requiere ingreso a Unidad de Cuidado Intensivo. Se documenta un neumotórax; se inicia manejo de oxígeno $(\mathrm{O} 2)$ y requiere punción torácica con posterior resolución y egreso sin complicaciones.

Los cambios fisiológicos en el momento del nacimiento deben ser entendidos por todo el personal que está a cargo de los recién nacidos, para poder anticipar las complicaciones que se presentan en los neonatos. Se debe hacer énfasis en la importancia de evitar el egreso temprano antes de las 24 horas, que eventualmente pueden llegar a tener consecuencias fatales.

Palabras Clave: Neumotórax espontáneo en neonatos; recién nacido; escape aéreo; fisiología pulmonar.

\footnotetext{
Abstract

Symptomatic spontaneous pneumothorax is a rare event in newborns. Although its course is benign and has spontaneous resolution in most cases, more aggressive interventions must be performed in some cases. There is no record of a case of spontaneous pneumothorax in the newborn in the
}

1 https://orcid.org/0000-00029276-8687 Médico-Pediatra Universidad del Sinú, estudiante de Epidemiología Universidad Autónoma de Bucaramanga. Pediatría Unidad Recién Nacido, Clínica Nueva de Cali.

${ }^{2}$ Médica-Neonatología Universidad del Valle. Coordinadora Unidad de Clínica Nueva de Cali.

${ }^{3}$ Pediatra Universidad del Valle, Clínica Nueva de Cali. Docente Universidad Javeriana.

${ }^{4}$ Pediatra Universidad del Valle. Clínica Nueva de Cali

\section{Autor de Correspondencia Jaime Alberto Urbiña Villarraga Correo electrónico: jaurbinav@hotmail.com}

Recibido: Octubre 52020

Aceptado: Octubre 302020 
Colombian literature, nor complete statistics of similar events of air leaks.

This is the case of a full-term newborn, male, with eutocic (normal) delivery and good adaptation at the time of birth, who develops severe respiratory distress over the hours that requires admission to the Intensive Care Unit. A pneumothorax is present; oxygen (O2) management is started, and he requires a thoracic puncture with subsequent resolution and discharge without complications.

All staff caring for newborns must understand physiological changes at the time of birth to anticipate complications in newborns. Emphasis should be placed on the importance of avoiding early discharge before 24 hours, which can eventually have fatal consequences.

Keywords: Spontaneous pneumothorax in neonates; newborn; air leaks; pulmonary physiology.

\section{Introducción}

El nacimiento es la etapa con más cambios drásticos en la vida de la persona; se sale de la comodidad del vientre materno a un ambiente que algunos pueden considerar hostil. Los grandes cambios que ocurren al nacer nos muestran la alta resistencia que presentamos en esta etapa de la vida: cambios hormonales, cardiovasculares, grandes cambios respiratorios y aumento de estímulos externos con una reactividad importante del sistema neurológico. Esto nos obliga como profesionales de la salud a conocer todos los cambios y posibles complicaciones que se pueden presentar en esta etapa.

\section{Reporte de caso}

Recién nacida de sexo femenino de 37 semanas de edad gestacional, madre de 38 años, G5P1C4, alto riesgo por edad materna y múltiples cirugías abdominales previas. Controles prenatales adecuados, infecciones TORCH (Toxoplasmosis, Rubéola Citomegalovirus, Herpes simple y VIH) negativo, cultivo recto vaginal negativo.

El nacimiento se produce por cesárea anterior, adaptación espontánea; se aplica profilaxis del recién nacido, APGAR (Activity, Pulse, Grimace, Appearence, Respiration por su sigla en inglés) de 9 al minuto, 9 a los 5 minutos y 10 a los 10 minutos. Medidas antropométricas con peso 2.550 gramos, talla: 49 centímetros, Perímetro Cefálico: 33.5 centímetros, perímetro del tórax: 30 centímetros, perímetro del abdomen: 28 centímetros. Examen físico sin hallazgos patológico, Silverman de 0/10 (examen que valora la dificultad respiratoria de un recién nacido). Se traslada junto a la madre, con evolución clínica satisfactoria, no se evidenció hipoglucemia en mediciones consecutivas; la madre refiere que hay succión al pecho, meconio y micción presentes.

Al momento del egreso, a las 24 horas de vida, se aprecia dificultad respiratoria en la recién nacida, consistente en quejido, aleto nasal, tirajes intercostales y subcostales, además de cianosis generalizada. Signos vitales: Frecuencia cardiaca 102 latidos por minuto, frecuencia respiratoria 44 respiraciones por minuto, saturación de O2 $75 \%$. Se inicia oxígeno y se traslada a la unidad de recién nacidos para evaluación y manejo. Al llegar se observa dificultad respiratoria importante, saturación de $\mathrm{O} 2$ del $84 \%$ sin oxígeno, se inicia cámara de Hood con fracción inspirada de oxigeno (FIO2) elevada, mejorando la saturación paulatinamente y el patrón respiratorio. Frecuencia cardiaca 171 latidos por minuto, frecuencia respiratoria 68 respiraciones por minuto, tensión arterial $61 / 31 \mathrm{~mm}$ de $\mathrm{HG}$, tensión arterial media de $51 \mathrm{~mm}$ de $\mathrm{Hg}$ y saturación de O2 96 $\%$ con $\mathrm{FiO} 2$ 0,6. Se solicitan paraclínicos; en estos no se aprecian alteraciones de línea roja ni blanca ni en los reactantes de fase aguda.

En los gases se identifica ligera hipoxemia y acidosis metabólica compensada (Tabla 1). Se realiza ecocardiograma que muestra foramen oval amplio vs comunicación interauricular (Tabla 2), radiografía de tórax (Figura 1A) donde se aprecia neumotórax izquierdo masivo. La recién nacida logra estabilización respiratoria con el inicio de O2. El manejo inicial es expectante; en el control radiológico hay disminución del neumotórax (Figura 1B) pero por persistencia de la dificultad respiratoria, se decide realizar punción para drenaje. En la radiografía subsiguiente se confirma resolución completa de neumotórax (Figura 1C). El manejo posterior consiste en destete y retiro del O2. Egresa sin complicaciones. 
Tabla 1. Resultados de los paraclínicos

\begin{tabular}{|ll|}
\hline \multicolumn{1}{|c|}{ Laboratorios } & \multicolumn{1}{c|}{ Resultados } \\
Hemoclasificación & Mamá A RH + // Bebé O RH + \\
TSH neonatal & $1,5 \mathrm{uIU} / \mathrm{mL}$ \\
Hemograma & \\
Leucocitos & $22300 \mathrm{uL}$ \\
Neutrófilos & $76 \%$ \\
Linfocitos & $21 \%$ \\
\hline Monocitos & $3 \%$ \\
Basofilos & $0 \%$ \\
Eosinofilos & $0 \%$ \\
\hline Hemoglobina & $15.9 \mathrm{gr} / \mathrm{dL}$ \\
\hline Hematocrito & $44.4 \%$ \\
Plaquetas & $282000 \mathrm{uL}$ \\
\hline Proteína C Reactiva & $0,97 \mathrm{mg} / \mathrm{L}$ \\
\hline Hemocultivos & Negativos a las $72 \mathrm{hrs}$ \\
Ionograma & Na:143mmol/L- K:5,02 mmol/L \\
Gases arteriales & $\mathrm{pH}: 7,41$-PCO2:26,6 -PO2:64,8 -HCO3: 16,1 BE: -6., \\
& PFI: 162 \\
\hline
\end{tabular}

Tabla 1. Imágenes diagnósticas

\begin{tabular}{|ll|}
\hline \multicolumn{1}{|c|}{ Ecografias } & \multicolumn{2}{c|}{ Reporte } \\
\hline Ecografia transfontanelar & Estudio sin alteraciones \\
\hline Ecocardiograma doppler & $\begin{array}{l}\text { Foramen oval amplio vs CIA 4mm; Ductus arterioso pequeño sin } \\
\text { repercusión; Función biventricular conservada. Sin datos de } \\
\text { hipertensión pulmonar. }\end{array}$ \\
\hline
\end{tabular}

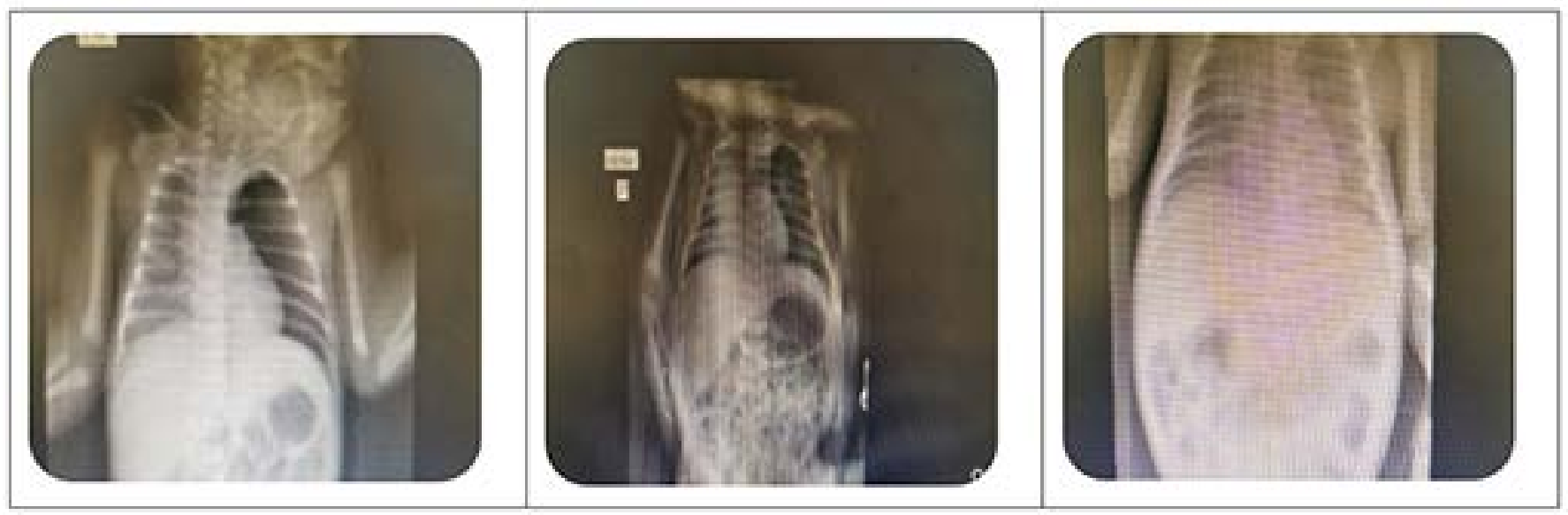

Figura 1. Imágenes diagnósticas de las radiografías de tórax. A. Neumotórax que ocupa casi la totalidad de hemitórax izquierdo; Hemitórax derecho con acentuación de trama peribronco vascular. No se visualiza neumotórax. Silueta cardíaca preservada. B. Se continúa observando neumotórax, pero menos significativo y expansión de parénquima pulmonar izquierdo. C. Radiografía posterior a punción de toracocentesis. Campos Pulmonares expandidos e Infiltrado intersticial difuso. 


\section{Fisiología respiratoria}

La respiración es el principal cambio al momento del nacimiento; recordemos que durante la etapa fetal los espacios aéreos pulmonares están llenos de líquido pulmonar fetal y no tienen función respiratoria, por lo cual al nacer se debe dar el paso de respiración liquida a respiración gaseosa. El líquido pulmonar es un ultrafiltrado del líquido intersticial conformado de la siguiente manera, cloro: $157 \mathrm{mEq} / \mathrm{L}$, sodio: $150 \mathrm{mE}$ q/L, potasio: $6.3 \mathrm{mEq} / \mathrm{L}$, bicarbonato: $2.8 \mathrm{mEq} / \mathrm{L}$, proteínas 0.027: $\mathrm{g} / \mathrm{dL}$, diferente a las características del líquido amniótico el cual está conformado así, cloro: $87 \mathrm{mEq} / \mathrm{L}$, sodio: $113 \mathrm{mEq} / \mathrm{L}$, potasio: $7.6 \mathrm{mE}$ q/L y proteínas 0.1: $\mathrm{g} / \mathrm{dL}$; permitiendo un $\mathrm{pH}$ de 6 en el líquido pulmonar.

La producción del líquido pulmonar fetal se realiza a partir del transporte activo de cloro plasmático, con intercambio de bicarbonato en la dirección opuesta por cambios en la presión hidrostática y osmótica, permitiendo el transporte iónico por uso de la bomba de NA/K ATPasa. Durante la gestación podemos encontrar un volumen entre 18 a $25 \mathrm{ml} / \mathrm{kg}$, disminuyendo de forma considerable al final de la gestación hasta $10 \mathrm{ml} / \mathrm{kg}$, para ser reabsorbido inmediatamente en el momento del nacimiento a través de un transporte activo de cloro a las vías linfáticas y sanguíneas. Pero este no es el único cambio, las presiones ejercidas sobre el pulmón con el primer llanto varían entre -40 y $+80 \mathrm{cmH} 20$ : con este cambio de presiones se puede ocasionar la ruptura alveolar y causar un neumotórax. El mantenimiento de la respiración se continua con diferentes estímulos que se presentan, como el frío, estimulo táctil, mediadores hormonales y si el clínico no conoce todos estos eventos. no puede comprender la importancia de este proceso $(1,2)$.

\section{Discusión}

El neumotórax se define como la presencia de aire entre la pleura parietal y la pleura visceral. Su aparición está relacionada con el entorno que rodea al paciente. La incidencia del neumotórax espontáneo en paciente pediátrico es de 3,4 por 10.000 ingresos pediátricos, pero en la etapa de recién nacido su frecuencia es mayor por los cambios rápidos de las altas presiones pulmonares que se presentan en las primeras respiraciones. El neumotórax espontáneo en el recién nacido puede pasar desapercibido en la mayoría con resolu- ción espontánea, pero en una mínima población puede ser sintomático y ocurre en un promedio de 0.05 a $1 \%$ y en algunos casos hasta el $2 \%$ en el recién nacido a término, según las diferentes series de estudios $(3,4)$. Desde el siglo XX se han reportado casos de neumotórax espontáneo. Silver en 1939 reportó seis casos incluyendo uno propio, en el que explica las causas de neumotórax espontáneo, asociado a patologías pulmonares con características de válvula única, causas explicadas para ese momento (5).

Tawil et al en 2004 en un estudio tomaron 80 recién nacidos desde 1991 al 2001, reportando la asociación del sexo masculino; el parto al vacío mostró una $\mathrm{p}=0.005$. El $80 \%$ de los pacientes no requirieron tratamiento invasivo. Durante la evaluación se encontraron cuatro pacientes con asociación de patología renal. Otra información de este estudio fue la ubicación del neumotórax, el hallazgo unilateral fue de $65 \%$ vs 27 $\%$ el bilateral. El lado derecho fue el más afectado con un $45 \%$ vs un $20 \%$ el izquierdo (6).

Otro reporte corresponde al de Katar et al en el 2006, con una cohorte pequeña de 11 pacientes. Encuentran que es más común en el sexo masculino, en los nacidos en vía cesárea, en los postérminos y los grandes para edad gestacional (7). Esto coincide con lo reportado por Gracia et al en el 2011, quienes asocian el neumotórax a la cesárea con una $\mathrm{p}=0.001$ y al recién nacido postérmino con una $\mathrm{p}=0.001$ (8). Además, el estudio de Katar et al, también encontró una relación con patología renal $18 \%$ con una $\mathrm{p}=0.47$, aunque reportan como casualidad por la cohorte pequeña y sugieren más estudios. No se realizó ecografía renal del volumen urinario del paciente.

En el caso que se presenta, un hallazgo en el ecocardiograma de comunicación interauricular CIA de 4 $\mathrm{mm}$ fue compatible con los hallazgos de Katar et al, que reportan la asociación con patología cardíaca en $6(54 \%)$ mostrando una asociación $(\mathrm{p}=0.012), 4$ con hallazgo de defecto de pared atrial, uno con defecto de pared y estenosis pulmonar y uno con defecto de pared y persistencia de ductus (7).

Kugleman et al en el 2003, muestran un reporte de dos familias para recopilación de cuatro casos que muestran la asociación de neumotórax familiar. En los casos reportados hablan de dos familias en que los recién nacidos son de sexo masculino, nacidos por cesáreas. Esta asociación tiene pocos reportes en la literatura, por lo que se debe realizar una asociación y estudios en los pacientes con antecedentes de hermanos que 
ingresan a unidad con diagnóstico de taquipnea respiratoria, ya que muchos clínicos por considerarla una patología benigna y de pronta resolución, no realizan radiografía de tórax y no se llega a la asociación de neumotórax familiar del recién nacido (9).

El tratamiento de los escapes aéreos se debe realizar en aquellos con clínica grave o en aquellos que no presenten mejoría. El tratamiento se puede realizar con (10):

1. Aspiración con aguja: se debe realizar en aquellos con neumotórax sin mayor compromiso o en aquellos neumotórax a tensión, que comprometan de forma significativa como medida de urgencia para evitar el colapso pulmonar.

2. Catéter de drenaje de pequeño calibre: puede ser técnica de punción directa tipo Pleurocath o por técnica de Seldinger para colocación de catéter "pig tail" (en forma de cola de cerdo).

3. Catéter grueso torácico.

El estudio de Simth et al en el 2011 siguió una cohorte por seis años de 4.906 recién nacidos, de los cuales 305 presentaron neumotórax, repartidos en 229 por causas secundarias y en 76 pacientes $(25 \%)$ correspondió a neumotórax espontáneos. Se registró un $71 \%$ de los pacientes sin requerimiento de tratamiento invasivo. Se concluye que el neumotórax espontáneo tiene un comportamiento diferente a los de causa secundaria, comportándose con un curso benigno (11).

Respecto a las diferentes técnicas, un metaanálisis reportado en Cochrane por Bruschettini et al en el 2019, compara la aspiración con aguja vs toracotomía en los recién nacidos en los que no se encontró diferencia en la mortalidad; el uso de aguja para aspiración disminuyó el requerimiento de tubo torácico (12).

\section{Conclusiones}

El neumotórax espontáneo es una entidad muy frecuente en el recién nacido, pero la elevada incidencia en pacientes asintomáticos hace que sea un evento casi imperceptible para el personal de salud. Los casos sintomáticos tienen una menor incidencia, pero en ocasiones pueden llegar a comprometer la integridad del recién nacido con gran importancia. Su curso es benigno con neumotórax espontáneo sin intervención invasiva, pero en ocasiones el clínico debe tener una respuesta rápida para evitar el colapso pulmonar. Conocer los cambios fisiológicos en el momento del nacimiento es fundamental para todos los que atienden recién nacidos.

Se recalca con este caso la importancia de la vigilancia conjunta con la madre y evitar en todo caso salidas tempranas (menos de 24 horas) para poder realizar las intervenciones rápidas y oportunas.

\section{Consentimiento para publicación}

Los autores declaran que se obtuvo consentimiento informado de los padres de la menor para la publicación del caso y las imágenes acompañantes. El consentimiento está disponible para el editor o los revisores si se requiere, por medio del autor de correspondencia.

\section{Conflicto de interés}

Los autores declaran que no hay ningún tipo de conflicto de intereses con respecto a la publicación de este artículo.

\section{Referencias}

1. Hillman N, Kallapur S, Jobe A. Physiology of Transition from Intrauterine to Extrauterine Life, Clin Perinatol. 2012;39: 769-783. doi: 10.1016/j. clp.2012.09.009.

2. Islas L. Líquido pulmonar fetal, Rev Med Hosp Gen Mex. 2006;69(4):221-225

3. Irastorza Terradillos IX, Landa Maya J, Gómez Cabanillas P. Neumotórax. An Pediatr. 2003;58(Supl 1):30-4

4. Hoyos A, Barotrauma y síndrome de perdida de aire. Pautas de recién nacidos, sexta edición. Editorial Celsus 2015, págs. 95 a 101.

5. Silver HB. Spontaneous pneumothorax in the newborn report of a case in which the mechanism was demonstrated. Am J Dis Chlid. 1939;57(4):907-915. doi:10.1001/archpedi.1939.01990040171013

6. Al Tawil K, Abu-Ekteish FM, Tamimi O, Al Hathal MM, Al Hathlol K, Abu Laimun B. Symptomatic spontaneous pneumothorax in term newborn infants. Pediatr Pulmonol. 2004;37(5):443-6. doi: $10.1002 /$ ppul.10447.

7. Katar S, Devecioğlu C, Kervancioğlu M, Ulkü R. Symptomatic spontaneous pneumothorax in term newborns. Pediatr Surg Int. 2006;22(9):7558. doi: 10.1007/s00383-006-1740-6. 
8. García R, Diez A, Aponte O, Pérez C, Gutiérrez L, García J. Influencia de la edad gestacional, el tipo de parto y la reanimación en el riesgo de neumotórax en neonatos mayores de 37 semanas. An Pediatr (Barc). 2014;80(3):138-143. doi: 10.1016/j.anpedi.2013.06.026

9. Kugelman A, Riskin A, Weinger-Abend M, Bader D. Familial neonatal pneumothorax associated with transient tachypnea of the newborn. Pediatr Pulmonol. 2003;36(1):69-72. doi: 10.1002/ ppul.10283.

10. Solís G, Mantecón L, Tratamiento de los escapes aéreos en neonatología. An Pediatr Con- tin. 2013;11(6):350-3. doi: 10.1016/S16962818(13)70158-6

11. Smith J, Schumacher RE, Donn SM, Sarkar S. Clinical course of symptomatic spontaneous pneumothorax in term and late preterm newborns: report from a large cohort. Am J Perinatol. 2011;28(2):163-8. doi: 10.1055/s-0030-1263300.

12. Bruschettini M, Romantsik O, Zappettini S, O'Donnell CP, Calevo MG. Needle aspiration versus intercostal tube drainage for pneumothorax in the newborn. Cochrane Database Syst Rev. 2019;2(2):CD011724. doi: 10.1002/14651858. CD011724.pub3 